\title{
Análise comparativa de plataformas baseadas em Cloud para o desenvolvimento de aplicações IoT
}

\author{
Lara Carolina Luciana e Oliveira ${ }^{1}$, Flávio de Oliveira Silva ${ }^{1}$ \\ ${ }^{1}$ Faculdade de Computação (FACOM) - Universidade Federal de Uberlândia (UFU) \\ Uberlandia - MG - Brazil \\ lara.carolinnaa@gmail.com, flavio@ufu.br
}

\begin{abstract}
The use of the Internet of Things (IoT) is growing, and cloud-based IoT platforms are a fundamental component of IoT applications. There are several platforms with different functionalities, capacities, prices, and limitations. In this scenario, how to choose the most suitable platform? What criteria to use in this choice? This work performs a comparative analysis of a set of IoT platforms with cloud-based architecture through a consistent and well-defined method. In addition to being more current than the literature currently available, the study considers a higher number of comparison criteria than the related research presenting valuable information for the community involved with IoT.
\end{abstract}

Resumo. O uso da Internet das Coisas (IoT) é crescente e plataformas de IoT baseadas na nuvem são um componente fundamental das aplicações de IoT. Existem diversas plataformas com diferentes funcionalidades, capacidades, preços e limitações. Neste cenário como escolher a plataforma mais adequada? Quais critérios utilizar nesta escolha? Este trabalho realiza análise comparativa de um conjunto de plataformas IoT com arquitetura baseada na nuvem através de um método consistente e bem definido. Além de ser mais atual que a literatura atualmente disponível, o trabalho considera um maior número de critérios de comparação que a literatura correlata apresentando informações valiosas para a comunidade envolvida com IoT.

\section{Introdução}

O crescente uso da Internet voltado para propósitos cada vez mais focados em auxiliar a vida dos usuários, resultou em um aumento significativo na variedade de dispositivos que interagem com ela. A sociedade atual vive em um mundo amplamente conectado através da integração de diferentes objetos com a Internet, culminando na era da Internet das Coisas (Internet of Things, IoT).

Neste contexto, as plataformas IoT exercem um importante papel. Além do desenvolvimento de aplicações, elas também oferecem ferramentas para coletar, armazenar, monitorar e analisar os dados gerados pelos dispositivos utilizados pela aplicação [Ismail et al. 2018]. Diante da importância da Computação em Nuvem para essas aplicações, grande parte das plataformas IoT possuem arquitetura baseada em nuvem, como é o caso das plataformas utilizadas como objeto de estudo neste trabalho.

É possível encontrar diversas plataformas IoT disponíveis para uso, cada uma contendo funcionalidades, capacidades, preços e limitações diferentes. Diante dessa variedade, o processo de escolha de uma plataforma que se adeque aos requisitos de uma aplicação pode ser confuso e exigir tempo. 
Neste cenário, surgem as seguintes questões: quais são as principais questões a se considerar no processo de escolha de uma plataforma? Quais são os principais critérios para avaliar plataformas IoT? De que maneira esses critérios se adequam a cada plataforma? Quais são as desvantagens e vantagens dessas plataformas? Essas e outras questões serão analisadas neste trabalho através da apresentação de uma análise comparativa com o intuito de auxiliar a escolha adequada de uma plataforma.

Este trabalho está organizado da seguinte forma: Seção 2 apresenta trabalhos relacionados. A Seção 3 apresenta o método utilizado para a realização da análise comparativa. A Seção 4 apresenta a análise comparativa. A Seção 5 faz uma discussão dos resultados. Finalmente a Seção 6 apresenta a conclusão e trabalhos futuros.

\section{Trabalhos Relacionados}

Esta seção apresenta os principais trabalhos de pesquisa relacionados ao tema aqui abordado, os quais motivaram ou contribuíram de alguma forma para a realização deste estudo.

O trabalho apresentado em [Ganguly 2016], fornece uma análise comparativa de 10 plataformas IoT Cloud. O autor propõe um conjunto de 20 requisitos que devem ser atendidos pelas plataformas. São selecionadas, a partir de pesquisas no Google, as plataformas que atendem pelo menos $60 \%$ dos requisitos contidos no conjunto definido. Além disso, recomendações e reconhecimentos, como prêmios e aprovações por empresas de pesquisa de mercado, também são considerados para a seleção das plataformas. $O$ autor desconsidera fatores como satisfação dos usuários e presença de mercado na seleção das plataformas para comparação. Além disso, também são desconsideradas plataformas desenvolvidas por grandes empresas, como Amazon e Google.

Na pesquisa apresentada em [Ray 2016], vinte e seis plataformas IoT Cloud são avaliadas. A autora separa as plataformas de acordo com a principal especialidade de cada uma e é apresentado um breve resumo das plataformas com o objetivo de ressaltar as principais características de cada uma delas. Além disso, são identificados os prós e contras de cada plataforma. A análise comparativa entre as plataformas é apresentada sob forma de uma tabela, utilizando como critérios avaliativos as especialidades usadas para separar as plataformas. Por fim, a autora identifica os principais problemas enfrentados pelas plataformas: padronização, heterogeneidade, gerenciamento de energia, tolerância à falhas, middleware e identidade de nós IoT.

O estudo realizado por [Sruthi and Kavitha 2016] visa fornecer uma visão geral sobre plataformas IoT a fim de auxiliar usuários na escolha da plataforma mais adequada para suas aplicações. É considerado um conjunto de 10 plataformas. Os autores fornecem um resumo das plataformas e definem cinco critérios para compará-las.

Em [Mineraud et al. 2016], os autores definem um conjunto de sete características que devem ser analisadas durante o processo de escolha de uma plataforma IoT. Com base neste conjunto, trinta e nove plataformas são avaliadas. Contudo, o foco deste trabalho está em identificar as principais falhas entre as plataformas selecionadas e fornecer orientações para lidar com os problemas identificados.

Em [Nakhuva and Champaneria 2015], são discutidas as principais características de 11 plataformas IoT, julgadas pelos autores como as mais populares, de forma a identificar as funcionalidades chaves de cada uma delas. A pesquisa apresentada por 
[Pires et al. 2015], busca discutir os principais requisitos para plataformas de middleware para IoT. Os autores abordam a arquitetura de referência do IoT-A, discutindo seus componentes e interações.

\section{Método de Trabalho}

O primeiro passo para este trabalho foi selecionar as plataformas para este estudo. De acordo com o panorama fornecido em [Postscapes 2019], há uma quantidade numerosa de plataformas IoT baseadas em Cloud disponíveis atualmente. Realizar uma análise comparativa de todas essas plataformas não seria viável devido a limites práticos e de tempo. Dessa forma, foram adotados critérios de maneira a selecionar dez plataformas para análise.

Um dos critérios de seleção adotados diz respeito à popularidade. A fim de obter tais plataformas, foi considerado o relatório produzido pela IoT Analytics, empresa que estuda tendências no mercado de internet das coisas, onde estão listadas as 25 plataformas IoT mais populares tendo como base a satisfação dos usuários [IoT-Analytics 2019]. Foi selecionado um subconjunto de 6 plataformas cloud-based: AWS IoT, Microsoft Azure IoT, Oracle IoT Cloud, Google Cloud ToT, Cisco Kinetic e IBM Watson IoT.

Além da popularidade, a característica open-source também foi considerada como critério de seleção. A pesquisa realizada pelo grupo BCG, apresentada em [Bhatia et al. 2017], buscou identificar os fatores mais atrativos em uma plataforma segundo a opinião de 300 desenvolvedores. Segundo a pesquisa, o fator mais atraente é a característica open-source. A fim de obter plataformas com tal característica, foi utilizado o panorama apresentado em [Postscapes 2019], onde estão listadas 4 plataformas open-source e cloud-based: ThingSpeak, Kaa, Macchina e SiteWhere.

\subsection{Definição dos conjuntos de métricas}

A fim de gerar as métricas utilizadas na análise comparativa foi realizada uma pesquisa de caráter exploratório acerca dos principais aspectos para avaliar uma plataforma IoT.

A maioria dos critérios encontrados nas fontes exploradas estão relacionados aos serviços e ferramentas que devem ser oferecidos pelas plataformas. É necessário que uma plataforma seja capaz de atender a um conjunto de funcionalidades essenciais a fim de possibilitar o provisionamento e gerenciamento das aplicações. Além das funcionalidades essenciais, é interessante considerar o suporte à tendências IoT inovadoras que podem ser vistas como fatores diferenciais entre as plataformas.

É também importante avaliar as plataformas sobre a perspectiva de características gerais como fornecimento de documentação, tipo de licença e oferecimento de suporte aos usuários. Características operacionais também devem ser consideradas como protocolos de comunicação suportados, SDKs fornecidos ou linguagens suportadas, suporte a dispositivos heterogêneos, entre outros. Outro grupo de características que deve ser avaliado diz respeito aos aspectos de segurança.

Considerando a revisão bibliográfica realizada foram determinados 26 critérios a fim de conduzir a análise comparativa entre as plataformas selecionadas, os quais são apresentados na Figura 1. 


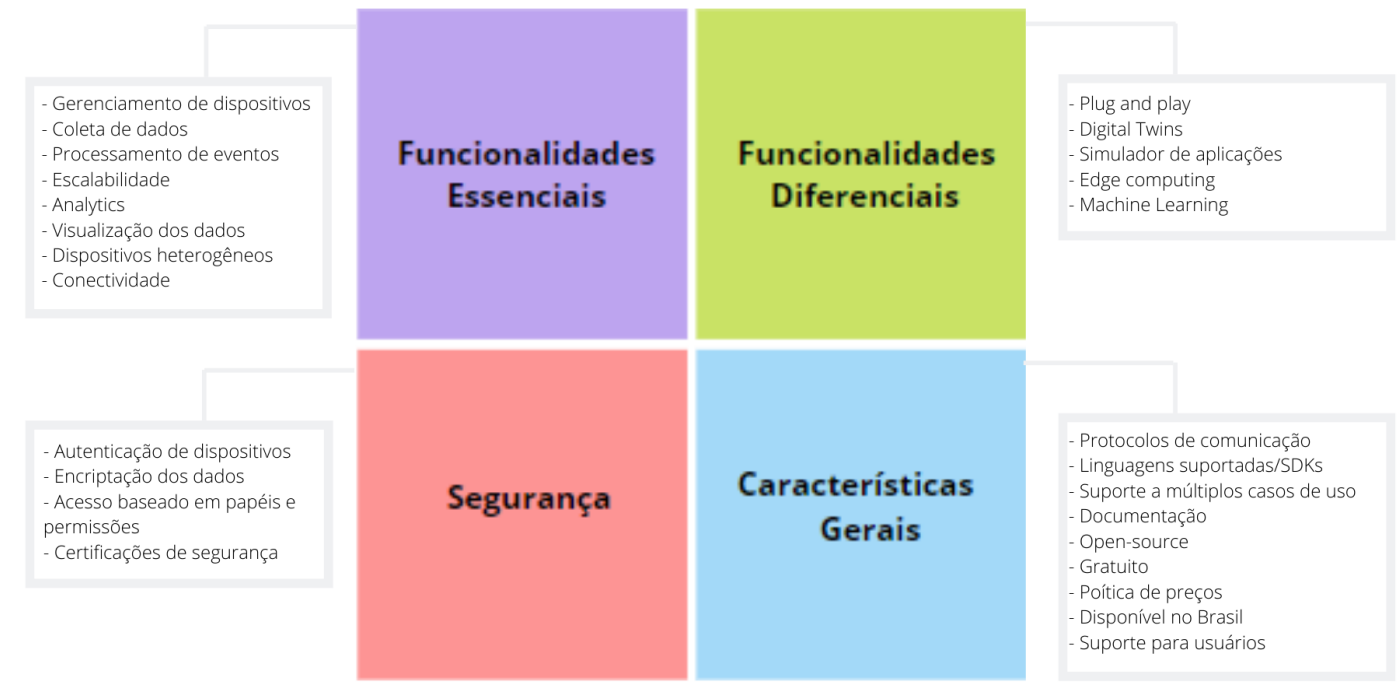

Figura 1. Conjuntos de métricas definidos para a análise comparativa

\subsection{Critérios de Pontuação}

A fim de auxiliar a interpretação dos resultados da análise comparativa foram definidos critérios de pontuação, os quais servirão para distribuir pontos entre as plataformas e classificá-las de acordo com a pontuação obtida. A métrica definida para verificar a política de preços adotada não será pontuada pois tal característica fica a critério e preferência do usuário. A característica geral chamada Disponível no Brasil foca na disponibilidade da infraestrutura da plataforma no Brasil.

As plataformas receberão 1 ponto caso um critério seja atendido, 0.5 pontos caso o critério seja parcialmente atendido e 0 pontos caso o mesmo não seja atendido. Todos os critérios serão pontuados de acordo com tal regra, exceto os seguintes:

- SDKs/Linguagens suportadas: a quantidade de pontos obtida será igual à quantidade de SDKs ou linguagens suportadas.

- Protocolos de comunicação: a quantidade de pontos obtida será igual à quantidade de protocolos suportados.

- Documentação: a plataforma receberá 1 ponto caso a documentação disponibilize, além de tutoriais, downloads de amostras de código de aplicações reais, 0.5 pontos caso forneça apenas tutoriais e 0 pontos caso não ofereça documentação.

Para os critérios SDKs/Linguagens suportadas e Protocolos de comunicação, o valor máximo obtido por uma plataforma será normalizado em um ponto e demais ajustados proporcionalmente. Assim todos os critérios terão o mesmo peso na avaliação.

\section{Análise Comparativa}

Esta seção apresenta a análise comparativa das plataformas selecionadas em diferentes tabelas baseando-se no conjunto de métricas definido na seção anterior.

As colunas podem ser preenchidas com símbolos ou informações, dependendo do critério. Caso uma plataforma atenda ao critério definido por uma coluna, a coluna é preenchida com o símbolo $\checkmark$, caso contrário, a coluna é preenchida com o símbolo $X$. Caso a plataforma atenda parcialmente ao critério, a coluna receberá o símbolo $\square$. 
A fim de verificar se uma plataforma atende ou não a um critério avaliativo, foram realizadas pesquisas exploratórias considerando a documentação disponibilizada e informações extraídas a partir de fontes secundárias, como sites e artigos.

Considerando o conjunto de critérios definidos para avaliar as funcionalidades essenciais, foi produzida a Tabela 1. Convém salientar que, para atribuir pontos às plataformas segundo o critério de Escalabilidade, foi considerado o estudo apresentado em [Hill 2019], que avalia as plataformas IoT com foco em critérios de escalabilidade. Já a análise comparativa de acordo com as funcionalidades vistas como diferenciais é apresentada na Tabela 2. A comparação das plataformas de acordo com os critérios definidos para analisar os aspectos relacionados à segurança é apresentada na Tabela 3. Finalmente, a partir dos critérios para analisar as características gerais foi produzida a Tabela 4.

Tabela 1. Comparação de acordo com as funcionalidades essenciais

\begin{tabular}{|c|c|c|c|c|c|c|c|c|c|c|}
\hline & AWS IoT & $\begin{array}{l}\text { Cisco } \\
\text { Kinetic }\end{array}$ & $\begin{array}{l}\text { Google } \\
\text { Cloud } \\
\text { IoT } \\
\end{array}$ & $\begin{array}{l}\text { IBM } \\
\text { Watson } \\
\text { IoT } \\
\end{array}$ & Kaa & Macchina & $\begin{array}{l}\text { Microsoft } \\
\text { Azure } \\
\text { IoT } \\
\end{array}$ & $\begin{array}{l}\text { Oracle } \\
\text { IoT } \\
\text { Cloud }\end{array}$ & SiteWhere & ThingSpeak \\
\hline $\begin{array}{l}\text { Gerenciamento } \\
\text { de dispositivos }\end{array}$ & $\checkmark$ & $x$ & $\checkmark$ & $\checkmark$ & $\checkmark$ & $\checkmark$ & $\checkmark$ & $\checkmark$ & $\checkmark$ & $x$ \\
\hline $\begin{array}{l}\text { Processamento } \\
\text { de eventos }\end{array}$ & $\checkmark$ & $\checkmark$ & $\checkmark$ & $\checkmark$ & $\checkmark$ & $\checkmark$ & $\checkmark$ & $\checkmark$ & $\checkmark$ & $\checkmark$ \\
\hline Coleta de dados & $\checkmark$ & $\checkmark$ & $\checkmark$ & $\checkmark$ & $\checkmark$ & $\checkmark$ & $\checkmark$ & $\checkmark$ & $\checkmark$ & $\checkmark$ \\
\hline Escalabilidade & $\checkmark$ & $\square$ & $\square$ & $\checkmark$ & $\square$ & $\square$ & $\checkmark$ & $\square$ & $x$ & $x$ \\
\hline Analytics & $\checkmark$ & $x$ & $\checkmark$ & $\checkmark$ & $\square$ & $x$ & $\checkmark$ & $\checkmark$ & $\square$ & $\checkmark$ \\
\hline $\begin{array}{l}\text { Visualização dos } \\
\text { dados }\end{array}$ & $\checkmark$ & $x$ & $\checkmark$ & $\checkmark$ & $\square$ & $\checkmark$ & $\checkmark$ & $\checkmark$ & $\square$ & $\checkmark$ \\
\hline $\begin{array}{ll}\text { Suporte } & \text { à } \\
\text { dispositivos } & \\
\text { heterogêneos } & \end{array}$ & $\checkmark$ & $\checkmark$ & $\checkmark$ & $\checkmark$ & $\checkmark$ & $x$ & $\checkmark$ & $\checkmark$ & $\checkmark$ & $\checkmark$ \\
\hline Conectividade & $\checkmark$ & $\checkmark$ & $\checkmark$ & $\checkmark$ & $\checkmark$ & $\checkmark$ & $\checkmark$ & $\checkmark$ & $\checkmark$ & $\checkmark$ \\
\hline Pontos & 8 & 4.5 & 7.5 & 8 & 6.5 & 5.5 & 8 & 7.5 & 6 & 6 \\
\hline
\end{tabular}

Tabela 2. Comparação de acordo com as funcionalidades diferenciais

\begin{tabular}{|c|c|c|c|c|c|c|c|c|c|c|}
\hline & AWS IoT & $\begin{array}{l}\text { Cisco } \\
\text { Kinetic }\end{array}$ & $\begin{array}{l}\text { Google } \\
\text { Cloud } \\
\text { IoT }\end{array}$ & $\begin{array}{l}\text { IBM } \\
\text { Watson } \\
\text { IoT }\end{array}$ & Kaa & Macchina & $\begin{array}{l}\text { Microsoft } \\
\text { Azure } \\
\text { IoT }\end{array}$ & $\begin{array}{l}\text { Oracle } \\
\text { IoT } \\
\text { Cloud }\end{array}$ & SiteWhere & ThingSpeak \\
\hline Plug-and-play & $x$ & $x$ & $\square$ & $\square$ & $x$ & $\checkmark$ & $\checkmark$ & $x$ & $x$ & $x$ \\
\hline $\begin{array}{l}\text { Digital Twins ou } \\
\text { Device Shadows }\end{array}$ & $\checkmark$ & $x$ & $x$ & $\checkmark$ & $\checkmark$ & $x$ & $\checkmark$ & $\checkmark$ & $x$ & $x$ \\
\hline $\begin{array}{ll}\text { Simulador } \\
\text { dispositivos }\end{array}$ & $\checkmark$ & $x$ & $x$ & $\checkmark$ & $x$ & $\checkmark$ & $\checkmark$ & $\checkmark$ & $x$ & $x$ \\
\hline Edge Computing & $\checkmark$ & $\checkmark$ & $\checkmark$ & $\checkmark$ & $x$ & $\checkmark$ & $\checkmark$ & $\checkmark$ & $x$ & $x$ \\
\hline $\begin{array}{l}\text { Machine Lear- } \\
\text { ning }\end{array}$ & $\checkmark$ & $x$ & $\checkmark$ & $\checkmark$ & $\checkmark$ & $x$ & $\checkmark$ & $\checkmark$ & $\checkmark$ & $\checkmark$ \\
\hline Pontos & 4 & 1 & 2.5 & 4.5 & 2 & 3 & 5 & 4 & 1 & 1 \\
\hline
\end{tabular}

Tabela 3. Comparação entre as plataformas de acordo com aspectos de segurança

\begin{tabular}{|l|c|c|c|c|c|c|c|c|c|}
\hline & AWS IoT & $\begin{array}{l}\text { Cisco } \\
\text { Kinetic }\end{array}$ & $\begin{array}{l}\text { Google } \\
\text { Cloud } \\
\text { IoT }\end{array}$ & $\begin{array}{l}\text { IBM } \\
\text { Watson } \\
\text { IoT }\end{array}$ & Kaa & Macchina & $\begin{array}{l}\text { Microsoft } \\
\text { Azure } \\
\text { IoT }\end{array}$ & $\begin{array}{l}\text { Oracle } \\
\text { IoT } \\
\text { Cloud }\end{array}$ & $\begin{array}{l}\text { SiteWhere } \\
\text { ThingSpeak }\end{array}$ \\
\hline $\begin{array}{l}\text { Autenticação de } \\
\text { dispositivos }\end{array}$ & $\checkmark$ & $\checkmark$ & $\checkmark$ & $\checkmark$ & $\checkmark$ & $x$ & $\checkmark$ & $\checkmark$ & $x$ \\
\hline $\begin{array}{l}\text { Suporte a } \\
\text { protocolos } \\
\text { criptográficos }\end{array}$ & $\checkmark$ & $\checkmark$ & $\checkmark$ & $\checkmark$ & $\checkmark$ & $\checkmark$ & $\checkmark$ & $\checkmark$ & $\checkmark$ \\
\hline $\begin{array}{l}\text { Acesso baseado } \\
\text { em papéis e per- } \\
\text { missões }\end{array}$ & $\checkmark$ & $\checkmark$ & $\checkmark$ & $\checkmark$ & $\checkmark$ & $\checkmark$ & $\checkmark$ & $\checkmark$ & $\checkmark$ \\
\hline $\begin{array}{l}\text { Certificações de } \\
\text { Segurança }\end{array}$ & $\checkmark$ & $\checkmark$ & $\checkmark$ & $\checkmark$ & $x$ & $x$ & $\checkmark$ & $\checkmark$ & $x$ \\
\hline Pontos & 4 & 4 & 4 & 4 & 3 & 2 & 4 & 4 & $x$ \\
\hline
\end{tabular}


Tabela 4. Comparação entre as plataformas de acordo características gerais

\begin{tabular}{|c|c|c|c|c|c|c|c|c|c|c|}
\hline & AWS IoT & $\begin{array}{l}\text { Cisco } \\
\text { Kinetic }\end{array}$ & $\begin{array}{l}\text { Google } \\
\text { Cloud } \\
\text { IoT }\end{array}$ & $\begin{array}{l}\text { IBM } \\
\text { Watson } \\
\text { IoT }\end{array}$ & Kaa & Macchina & $\begin{array}{l}\text { Microsoft } \\
\text { Azure } \\
\text { IoT }\end{array}$ & $\begin{array}{l}\text { Oracle } \\
\text { IoT } \\
\text { Cloud }\end{array}$ & SiteWhere & ThingSpeak \\
\hline $\begin{array}{l}\text { Protocolos } \\
\text { de } \\
\text { comunicação }\end{array}$ & $\begin{array}{l}\text { MQTT, } \\
\text { HTTPS, } \\
\text { MQTT } \\
\text { sobre } \\
\text { WebSoc- } \\
\text { ket }\end{array}$ & Independente & $\begin{array}{l}\text { MQTT e } \\
\text { HTTP }\end{array}$ & $\begin{array}{l}\text { MQTT e } \\
\text { HTTP }\end{array}$ & $\begin{array}{l}\text { MQTT } \\
\text { e CoAP }\end{array}$ & $\begin{array}{l}\text { MQTT } \\
\text { e Web- } \\
\text { Sockets }\end{array}$ & $\begin{array}{l}\text { MQTT, } \\
\text { MQTT } \\
\text { so- } \\
\text { breWeb- } \\
\text { Sockets, } \\
\text { AMQP, } \\
\text { AMQP } \\
\text { sobre } \\
\text { Web- } \\
\text { Sockets, } \\
\text { HTTPS }\end{array}$ & $\begin{array}{l}\text { HTTP } \\
\text { e } \\
\text { MQTT }\end{array}$ & $\begin{array}{l}\text { MQTT, } \\
\text { AMQP, } \\
\text { Web- } \\
\text { Sockets, } \\
\text { STOMP }\end{array}$ & MQTT \\
\hline $\begin{array}{l}\text { Linguagens } \\
\text { suporta- } \\
\text { das/SDKs }\end{array}$ & $\begin{array}{l}\text { Arduino } \\
\text { Yún, An- } \\
\text { droid, C, } \\
\text { C++, iOS, } \\
\text { Java, Ja- } \\
\text { vaScript, } \\
\text { Python }\end{array}$ & $x$ & $\begin{array}{l}\text { Go, Java, } \\
\text {.NET, } \\
\text { Node.js, } \\
\text { PHP, } \\
\text { Python, } \\
\text { Ruby }\end{array}$ & $\begin{array}{l}\text { C, Java, } \\
\text { Node.js, } \\
\text { Python }\end{array}$ & $\begin{array}{l}\mathrm{C}, \mathrm{C}++, \\
\text { Java, } \\
\text { Objective } \\
\mathrm{C}\end{array}$ & $\begin{array}{l}\mathrm{C}++\mathrm{e} \\
\text { JavaS- } \\
\text { cript }\end{array}$ & $\begin{array}{l}\text {.NET, } \\
\text { C, Java, } \\
\text { Node.js, } \\
\text { Python, } \\
\text { iOS }\end{array}$ & $\begin{array}{l}\text { Java, } \\
\text { JavaS- } \\
\text { cript, } \\
\text { An- } \\
\text { droid, } \\
\text { C, } \\
\text { iOS }\end{array}$ & $\begin{array}{l}\text { Android, } \\
\text { iOS }\end{array}$ & $x$ \\
\hline $\begin{array}{l}\text { Suporte à } \\
\text { múltiplos } \\
\text { casos de uso }\end{array}$ & $\checkmark$ & $\checkmark$ & $\checkmark$ & $\checkmark$ & $\checkmark$ & $\checkmark$ & $\checkmark$ & $\checkmark$ & $\checkmark$ & $\checkmark$ \\
\hline Documentação & $\checkmark$ & $\sqrt{ }$ & $\checkmark$ & $\sqrt{ }$ & $\checkmark$ & $\sqrt{ }$ & $\checkmark$ & $\checkmark$ & $\sqrt{ }$ & $\checkmark$ \\
\hline Open-source & $x$ & $x$ & $x$ & $x$ & $\checkmark$ & $\checkmark$ & $x$ & $x$ & $\checkmark$ & $\checkmark$ \\
\hline Gratuita & $\square$ & $x$ & $\square$ & $\square$ & $\checkmark$ & $\square$ & $\square$ & $x$ & $\sqrt{ }$ & $\square$ \\
\hline $\begin{array}{l}\text { Política de } \\
\text { Preços }\end{array}$ & $\begin{array}{l}\text { Baseia- } \\
\text { se nos } \\
\text { serviços } \\
\text { contra- } \\
\text { tados e } \\
\text { utilização. } \\
12 \text { meses } \\
\text { gratui- } \\
\text { tos com } \\
\text { limitações }\end{array}$ & $\begin{array}{l}\text { Baseia- } \\
\text { se nos } \\
\text { produtos } \\
\text { contrata- } \\
\text { dos. Não } \\
\text { possui } \\
\text { versão } \\
\text { gratuita }\end{array}$ & $\begin{array}{l}\text { Baseia- } \\
\text { se no } \\
\text { volume } \\
\text { de dados } \\
\text { mensal } \\
\text { trocado } \\
\text { entre os } \\
\text { disposi- } \\
\text { tivos e } \\
\text { a plata- } \\
\text { forma }\end{array}$ & $\begin{array}{l}\text { Baseia- } \\
\text { se no nro } \\
\text { de dispo- } \\
\text { sitivos, } \\
\text { volume } \\
\text { e tráfego } \\
\text { de da- } \\
\text { dos. } \\
\text { Nível } \\
\text { gratuito } \\
\text { com } \\
\text { limitações }\end{array}$ & Gratuito & $\begin{array}{l}\text { Planos } \\
\text { de assi- } \\
\text { natura } \\
\text { men- } \\
\text { sais. } \\
\text { Possui } \\
\text { ní1vel } \\
\text { gratuito } \\
\text { com } \\
\text { limitações }\end{array}$ & $\begin{array}{l}\text { Baseia- } \\
\text { se nos } \\
\text { serviços } \\
\text { contra- } \\
\text { tados. } \\
\text { Cada } \\
\text { serviço } \\
\text { tem sua } \\
\text { própria } \\
\text { políitica } \\
\text { de } \\
\text { preços. } \\
12 \text { meses } \\
\text { gratui- } \\
\text { tos com } \\
\text { limitações. }\end{array}$ & $\begin{array}{l}\text { Planos } \\
\text { de as- } \\
\text { sina- } \\
\text { tura. } \\
\text { Não } \\
\text { possui } \\
\text { versão } \\
\text { gra- } \\
\text { tuita }\end{array}$ & Gratuito & $\begin{array}{l}\text { Planos de } \\
\text { assinatura. } \\
\text { Possui } \\
\text { versão } \\
\text { gratuita } \\
\text { com } \\
\text { limitações }\end{array}$ \\
\hline $\begin{array}{l}\text { Disponível } \\
\text { no Brasil }\end{array}$ & $\square$ & $\checkmark$ & $x$ & $\checkmark$ & $\checkmark$ & $\checkmark$ & $\square$ & $\square$ & $\checkmark$ & $\checkmark$ \\
\hline $\begin{array}{l}\text { Suporte para } \\
\text { usuários }\end{array}$ & $\checkmark$ & $\checkmark$ & $\checkmark$ & $\checkmark$ & $\checkmark$ & $\checkmark$ & $\checkmark$ & $\checkmark$ & $\checkmark$ & $\checkmark$ \\
\hline $\begin{array}{l}\text { Pontuação } \\
\text { Normalizada }\end{array}$ & 5.60 & 4.90 & 4.78 & 5.40 & 6.90 & 6.15 & 5.75 & 4.53 & 7.05 & 5.70 \\
\hline
\end{tabular}

\section{Discussão dos Resultados}

A Tabela 5 apresenta um ranking que foi construído a partir dos critérios de pontuação apresentados na Seção 3.2. A plataforma IoT que atendeu de forma mais satisfatória ao conjunto de critérios avaliativos foi a Microsoft Azure IoT, a qual ultrapassou a pontuação final da plataforma IBM Watson IoT por menos de um um ponto. As plataformas IBM Watson IoT, AWS IoT e Oracle Cloud IoT obtiveram pontuações próximas, sendo seguidas pela solução oferecida pelo Google. A diferença de pontuação entre plataforma que está na segunda posição (IBM Watson IoT) e a terceira (AWS IoT) foi de apenas 0.30 pontos. A adequação de tais plataformas em relação aos critérios também pode ser considerada satisfatória, indicando que as mesmas oferecem um rico conjunto de serviços.

A sexta posição na classificação foi ocupada pela plataforma Kaa IoT, seguida pelas plataformas Macchina.io, SiteWhere, Cisco Kinetic e ThingSpeak. Contudo, é importante salientar que, ainda assim, tais plataformas atendem a maior parte dos critérios avaliativos. 
Tabela 5. Classificação das plataformas

\begin{tabular}{|c|l|c|}
\hline Posição & \multicolumn{1}{|c|}{ Plataforma } & Pontuação \\
\hline 1 & Microsoft Azure IoT & 22.75 \\
\hline 2 & IBM Watson IoT & 21.90 \\
\hline 3 & AWS IoT & 21.60 \\
\hline 4 & Oracle Cloud IoT & 20.00 \\
\hline 5 & Google Cloud IoT & 18.80 \\
\hline 6 & Kaa IoT & 18.40 \\
\hline 7 & Macchina.io & 16.65 \\
\hline 8 & SiteWhere & 16.05 \\
\hline 9 & Cisco Kinetic & 14.40 \\
\hline 10 & ThingSpeak & 13.70 \\
\hline
\end{tabular}

É possível perceber que as plataformas open-source ocupam as posições inferiores no ranking, com exceção da plataforma Cisco Kinetic, a qual não apresenta a característica open-source.

Convém salientar que o fato de uma plataforma ocupar posições inferiores na classificação não indica que a mesma não possa oferecer uma solução satisfatória para uma determinada aplicação. É possível que uma aplicação IoT não necessite de uma plataforma que possua todas as características consideradas na pontuação.

A fim de estabelecer uma comparação entre as abordagens adotadas pelos trabalhos relacionados apresentados na Seção 2 e este estudo, alguns parâmetros foram considerados para realizar uma breve análise comparativa, apresentados na Tabela 6 .

Tabela 6. Comparação entre os trabalhos relacionados

\begin{tabular}{|c|c|c|c|c|}
\hline Trabalho & Critérios de Seleção & Plataformas & Critérios & Ano \\
\hline [Ganguly 2016] & $\begin{array}{l}\text { Adesão a pelo menos } 60 \% \text { dos requisitos listados pelo autor } \\
\text { e pelo menos uma recomendação ou reconhecimento }\end{array}$ & 10 & 21 & 2016 \\
\hline [Ray 2016] & Não há & 26 & 10 & 2016 \\
\hline [Sruthi and Kavitha 2016] & Não há & 10 & 5 & 2016 \\
\hline [Mineraud et al. 2016] & Não há & 39 & 7 & 2016 \\
\hline [Nakhuva and Champaneria 2015] & Popularidade & 11 & 11 & 2015 \\
\hline [Pires et al. 2015] & Não há & 11 & 8 & 2015 \\
\hline Este trabalho & Popularidade (6 plataformas) e Open-source (4 plataformas) & 10 & 26 & 2019 \\
\hline
\end{tabular}

A partir das tabelas construídas, é possível perceber que um dos principais diferenciais deste trabalho diz respeito a quantidade de critérios avaliativos utilizados na comparação com trabalhos encontrados na literatura.

É importante salientar que plataformas open-source como SiteWhere e Macchina.io não foram consideradas em nenhum dos trabalhos correlatos. Além disso, as plataformas aqui consideradas que também foram analisadas por outro trabalhos, foram comparadas neste trabalho sob perspectivas diferentes, ou seja, considerando critérios distintos. Isso mostra que este trabalho trouxe novas informações e resultados até mesmo para as plataformas já analisadas em outros estudos.

Convém salientar também a importância da data de publicação deste tipo de estudo. Pesquisas mais antigas podem não retratar as características atuais das plataformas, já que as mesmas estão em constante aprimoramento, correções e adição de funcionalidades. Dessa forma, é necessário que o público deste tipo de pesquisa opte por trabalhos mais recentes. Portanto, o fato de este trabalho ser o mais recente em relação aos trabalhos relacionados, mostra que foram consideradas informações e tendências mais atuais. 


\section{Conclusão e Trabalhos Futuros}

A utilização de plataformas pode ser vista como um requisito para o desenvolvimento de aplicações IoT. Diante da variedade de plataformas IoT Cloud disponíveis atualmente, podem surgir dificuldades no processo de definição de uma plataforma para utilização.

Considerando a análise comparativa desenvolvida e a apresentação dos resultados obtidos, pode-se concluir que as plataformas analisadas possuem suas próprias vantagens e desvantagens. Contudo, observamos que as plataformas que mais se adequaram ao conjunto de critérios definidos foram as soluções oferecidas pela Microsoft e AWS. Tais plataformas apresentaram pontuações satisfatórias em todos os conjuntos de critérios.

Foi possível observar que as plataformas open-source apresentaram menores pontuações, principalmente em relação as funcionalidades diferenciais, o que leva a concluir que estas plataformas geralmente fornecem apenas as funcionalidades básicas.

Um trabalho futuro seria a análise comparativa da performance das plataformas a partir de simulações a fim de identificar quais são mais eficientes quando expostas a diferentes situações de uso.

\section{Referências}

Bhatia, A., Yusuf, Z., Ritter, D., and Hunke, N. (2017). Who will win the iot platform wars? BCG Perspectives.

Ganguly, P. (2016). Selecting the right iot cloud platform. In 2016 International Conference on Internet of Things and Applications (IOTA), pages 316-320. IEEE.

Hill, S. (2019). Scalable iot platforms. Master's thesis.

IoT-Analytics (2019). The 25 best iot platforms 2019 - based on customer reviews.

Ismail, A. A., Hamza, H. S., and Kotb, A. M. (2018). Performance evaluation of open source iot platforms. In 2018 IEEE Global Conference on Internet of Things (GCIoT), pages $1-5$. IEEE.

Mineraud, J., Mazhelis, O., Su, X., and Tarkoma, S. (2016). A gap analysis of internetof-things platforms. Computer Communications, 89:5-16.

Nakhuva, B. and Champaneria, T. (2015). Study of various internet of things platforms. International Journal of Computer Science \& Engineering Survey, 6(6):61-74.

Pires, P. F., Delicato, F., Batista, T., Barros, T., Cavalcante, E., and Pitanga, M. (2015). Plataformas para a internet das coisas. Minicursos SBRC-Simpósio Brasileiro de Redes de Computadores e Sistemas Distribuídos.

Postscapes (2019). Iot cloud platform landscape.

Ray, P. P. (2016). A survey of iot cloud platforms. Future Computing and Informatics Journal, 1(1-2):35-46.

Sruthi, M. and Kavitha, B. (2016). A survey on iot platform. International Journal of Scientific Research and Modern Education (IJSRME), ISSN (online), pages 24555630 . 